\title{
Feasibility Study of High-Performance Implantable Stimulation Electrode with Nanocomposite Gel Coating as a Brain-Machine Interface Device
}

\author{
Hiroyuki Tashiro, ${ }^{1,2^{*}}$ Yasuo Terasawa, ${ }^{2,3}$ Kazutoshi Haraguchi, ${ }^{4}$ \\ Koji Osawa, ${ }^{3}$ Toshihiko Noda, ${ }^{2}$ Takashi Tokuda, ${ }^{2}$ and Jun Ohta ${ }^{2}$ \\ ${ }^{1}$ Kyushu University, 3-1-1 Maidashi, Higashi-ku, Fukuoka 812-8582, Japan \\ ${ }^{2}$ Nara Institute of Science and Technology, 8916-5 Takayama, Ikoma, Nara 630-0192, Japan \\ ${ }^{3}$ Nidek Co., Ltd., 13-2 Hama-cho, Gamagori, Aichi 443-0036, Japan \\ ${ }^{4}$ Nihon University, 1-2-1 Izumi-cho, Narashino, Chiba 275-8575, Japan
}

(Received March 14, 2016; accepted June 22, 2016)

Keywords: stimulation electrode, hydrogel, NC gel, BMI, DNI, visual prosthesis

The brain-machine interface (BMI) or direct neural interface (DNI) has been intensively investigated for patients with physical function disorders. These interfaces are direct communication pathways between the brain and an external device. Devices for BMI restore physical function in patients after refractory nerve injury. A general technique for transmitting information from a machine to a living body is electrical stimulation with metal electrodes. For transmitting more information, it is necessary to increase the electrode density by miniaturizing the size of the stimulation electrode. However, such miniaturization may deteriorate the performance of the maximum injectable charge without causing tissue damage, especially for charge injection in vivo. To address this issue, we have proposed an implantable stimulation electrode with a hydrophilic gel coating and fabricated platinum (Pt) electrodes covered with a nanocomposite (NC) gel. The performance of the Pt electrode with an NC gel coating is confirmed to be equal to that of electrodes without a gel in vitro. If the charge injection capacity of the electrode covered with the NC gel maintains this value via the absorption of extracellular fluid and the existence of sufficient ions and water around the electrode surface, the performance of the electrode in a living body could increase. The results of the in vitro evaluation of the fabricated electrode show good performance and suggest the enhancement of the in vivo performance.

\section{Introduction}

The brain-machine interface (BMI) and direct neural interface (DNI) have been under intense investigation. These interfaces are direct communication pathways between the brain and an external device. The purpose of BMI research and development has focused primarily on neural prosthetics applications that aim at restoring damaged hearing, sight, and motor function. Neural prostheses are artificial devices that replace the function of an impaired nervous system, brain, or sensory organs. ${ }^{(1)}$ The most widely used neural prosthetic device is the cochlear implant, which has been implanted in approximately 324200 people worldwide as of 2012.(2)

"Corresponding author: e-mail: htashiro@med.kyushu-u.ac.jp 
There are also several neural prosthetic devices that aim to restore vision, including artificial retinas. We have been developing retinal prosthesis devices using the suprachoroidal-transretinal stimulation (STS) approach with the 49-channel (ch) electrode array for clinical trial. ${ }^{(3)}$ In this way, the general technique for transmitting information from the machine to the living body is electrical stimulation with a metal electrode. To further improve the quality of life of patients, it is necessary to increase the resolution of the electrode array. For other applications, it is expected to provide high densities of microelectrodes with improved spatial selectively and also safe stimulation. ${ }^{(4)}$ General methods for improving the electrode performance involve increasing the real surface area of the electrode relative to its geometric surface. ${ }^{(5,6)}$ However, there is a tradeoff between increasing the surface area and high densification of the electrode array. Therefore, another strategy for increasing the electrode density is required.

Excess charge injection via metal electrodes provokes local $\mathrm{pH}$ exchange via electrolysis as well as the production of electrolytic deposits. These phenomena occur when the injection charge exceeds the critical value called the charge injection capacity (CIC). Previous studies have shown that the CIC of the stimulation electrode in vivo is considerably smaller than the value in vitro. ${ }^{(7)}$ Some reasons for the decrease in the CIC are ionic depletion required for electrochemical reaction, a minor amount of water, and low perfusion of the electrolysis solution. If sufficient amounts of ions and water exist around the stimulation electrode, the CIC in an in vivo situation could be expected to increase. Therefore, we invented a stimulation electrode in which the surface is coated with a highly hydrous gel. Hydrogels provide minimal hindrance to the ions required for capacitive charge transfer in vivo. On the basis of the same principle, Ref. 4 reported Pt electrodes coated with polyvinyl alcohol (PVA) and polyethylene glycol (PEG) for implants. However, the hydrogels that are as soft as the tissue present difficulties during implantation. ${ }^{(8)}$ In order to address this issue, we propose the hydrogel-coated electrode using a nanocomposite (NC) gel with high mechanical property as the coating material. The objective of this study is to confirm the feasibility of improving the performance of implantable stimulation electrodes by coating their surfaces with the $\mathrm{NC}$ gel and to clarify the characteristics of the stimulation electrode with the NC gel coating in vitro.

\section{Materials and Methods}

\subsection{Hydrogel}

$\mathrm{NC}$ gels were used for the hydrogels owing to their high mechanical properties and good biocompatibility. ${ }^{(9)}$ The NC gels were synthesized by free radical polymerization of $\mathrm{N}, \mathrm{N}$ dimethylacrylamide (DMAA) in the presence of exfoliated inorganic clay (synthetic hectorite: Laponite ${ }^{\circledR}$ XLG, Rockwood Ltd.) in a potassium persulfate (KPS)-initiated aqueous system. Here, exfoliated clay nanosheets act as multi-functional cross-linkers for poly-DMAA (PDMAA). The concentrations of clay (hectorite) and DMAA in the aqueous reaction solution were $2.3 \mathrm{wt} \%$ and 1 $\mathrm{mol} / \mathrm{L}-\mathrm{H}_{2} \mathrm{O}$, respectively. Because the hectorite nanosheets have a negative charge on the surface, the NC gels have a tendency to incorporate more cations. ${ }^{(10)}$ Therefore, organic cross-linked (OR) gels with electrically neutral cross-linkers were used as a reference. The OR gels were prepared by the same procedure as that for the $\mathrm{NC}$ gel, except for the use of an organic cross-linker $\left[N, N^{\prime}\right.$ methylenebis (acrylamide) (BIS)] instead of hectorite. Moreover, the NC gels exhibited high 
adhesiveness to metal because of the unique polymer (PDMAA)-clay network structure. The cutting surface of the $\mathrm{NC}$ gel has self-adhesiveness to metal by electrostatic force owing to the negatively charged hectorite. Hence, the NC gels are appropriate for coating material applications in metal stimulation electrodes. These gels were soaked in $0.01 \mathrm{~mol} / \mathrm{L}$ phosphate buffered saline (PBS) (-) (164-84541, Wako) for over $24 \mathrm{~h}$. Then, test pieces were cut to a size of $3 \mathrm{~cm}$ square and $5 \mathrm{~mm}$ thickness. The electron transfer occurs extremely near the electrode surface, and the thickness of the electrical double layer is less than $10 \mathrm{~nm}$. ${ }^{(11)}$ Additionally, all measurements were performed within the electron transfer limitation. Therefore, the results were not dependent on the size of the gels.

The $\mathrm{pH}$ of these gels was measured with a surface $\mathrm{pH}$ electrode (0040-10D, HORIBA) and $\mathrm{pH}$ meter (D-51, HORIBA). The water content ratio of the gels was measured with a heat-drying-type moisture meter (MX-50, ANDO).

\subsection{Evaluation electrode}

Three Pt wire electrodes with diameters (dia.) of $200 \mu \mathrm{m}$ (PT-351265, Nilaco) were used for cyclic voltammetry (CV). The electrodes were placed in the hydrogels with an insertion depth of $1 \mathrm{~cm}$. Then, the hydrogels were dipped in $0.01 \mathrm{~mol} / \mathrm{L}$ PBS (-) during the CV measurements [Fig. 1(a)]. For measurement without hydrogels, the dipping depth in the PBS solution was $1 \mathrm{~cm}$.

In the other measurements, three Pt disk electrodes of $10 \mathrm{~mm}$ dia. and $100 \mu \mathrm{m}$ thickness (PT-356320, Nilaco) were used (Fig. 2). Electrochemical measurements were performed with the disk electrodes attached to the hydrogels on an acrylic board with a $12 \mathrm{~mm}$ opening area [Fig. 1 (b)]. Furthermore,

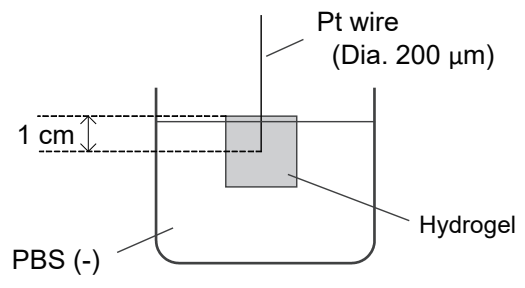

(a)

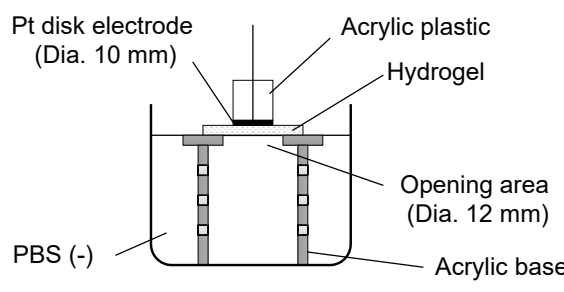

(b)

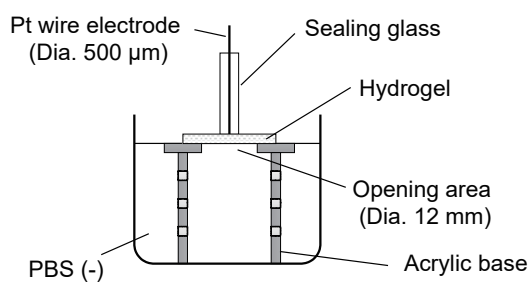

(c)

Fig. 1. Placement of the Pt evaluation electrodes. (a) Pt wire electrode in CV. (b) Pt disk electrode. (c) Glasssealed Pt electrode.

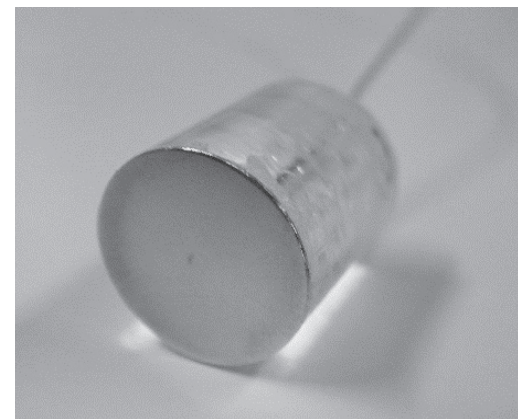

Fig. 2. Pt disk electrode of $10 \mathrm{~mm}$ dia. 
three glass-sealed Pt wire (PT-351381, Nilaco) electrodes of $500 \mu \mathrm{m}$ dia. were used to evaluate the difference in the electrochemical characteristics owing to the small electric double-layer capacitance of the microelectrodes [Figs. 1(c) and 3]. A silver-silver chloride $(\mathrm{Ag} / \mathrm{AgCl})$ electrode (RE-1C, BAS) was used as a reference electrode (RE), and a Pt wire electrode of $500 \mu \mathrm{m}$ dia. and sufficient length (PT-351385, Nilaco) was used as a counter electrode (CE) for all measurements.

\section{$2.3 \mathrm{CV}$}

The CV system with a potentiostat (AUTOLAB PGSTAT32, Metrohm), shown in Fig. 4, was constructed to confirm whether the voltage range that generated a Faradaic reaction was changed by the presence of the hydrogel coating. The evaluation electrodes were connected to the terminal of the working electrode (WE). After electrochemical cleanings were performed by applying force direct current voltage between -1 and $+1 \mathrm{~V}$ for $3 \mathrm{~s}$, CV was performed using a scan range from -0.85 to $+0.9 \mathrm{~V}$ at a scan rate of $0.1 \mathrm{~V} / \mathrm{s}$ and 10 scans. The water windows were determined from the results of $\mathrm{CV}$, and the differential was evaluated between the conditions of "inserted in the $\mathrm{NC}$ gel" (NC gel), "inserted in the OR gel" (OR gel), and "without gel" (PBS).

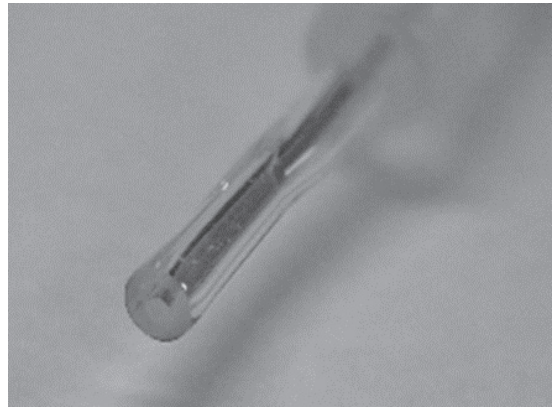

(a)

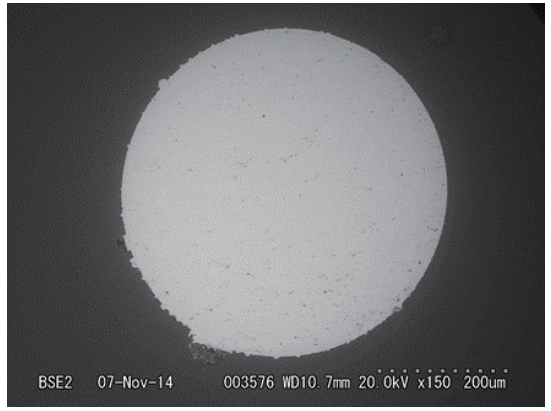

(b)

Fig. 3. Glass-sealed Pt wire electrode of $500 \mu \mathrm{m}$ dia. (a) Optical micrograph. (b) Scanning electron microscopy image.

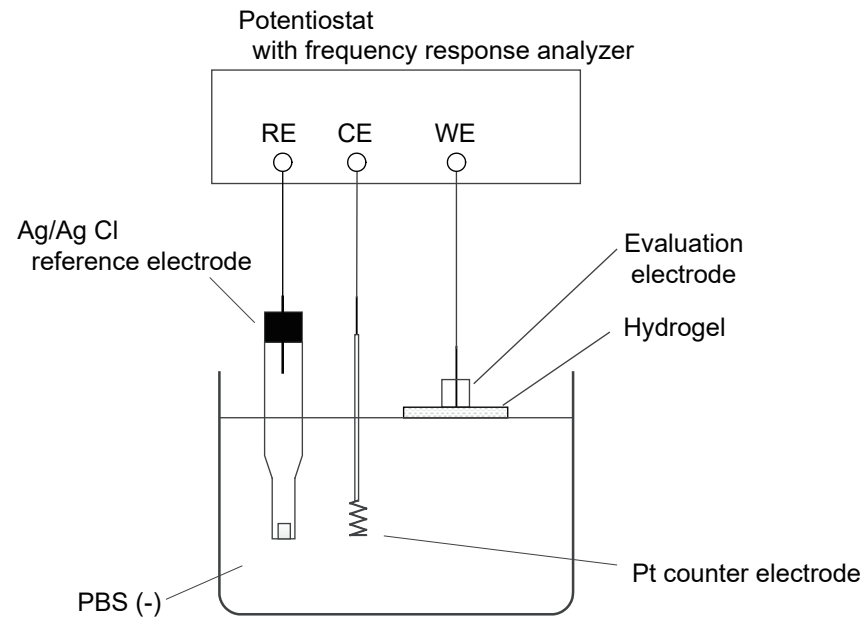

Fig. 4. Components of the CV and EIS system. 


\subsection{Electrochemical impedance spectroscopy (EIS)}

EIS was performed using the same CV system (Fig. 4). The scan range with the Pt disk electrodes was from $0.1 \mathrm{~Hz}$ to $100 \mathrm{kHz}$, while that with the glass-sealed Pt electrodes was from $100 \mathrm{~Hz}$ to $100 \mathrm{kHz}$. The applied voltages were $10 \mathrm{mV}_{\mathrm{rms}}$ constant-voltage sine waves. The circuit constants of the equivalent circuit shown in Fig. 5 were calculated using the EIS results with an equivalent circuit simulator (NOVA2, Metrohm). The solution resistance $R_{\text {sol }}$ is the bulk resistance of the solution itself. The charge transfer resistance $R_{\mathrm{ct}}$ defines the difficulty of electron transfer between the electrode surface and the solution. The constant-phase element (CPE) was used for the definition of double-layer capacitance that appeared at the interface between the electrode and the liquid electrolyte instead of simple capacitance because a complex solution such as PBS has several processes with differential time constants. Additionally, the CPE defines the nonuniformity and roughness of the electrode surface. The impedance of $\mathrm{CPE} Z_{\mathrm{CPE}}$ is represented by

$$
Z_{\mathrm{CPE}}=\frac{1}{(j \omega)^{n} Y_{0}}
$$

where CPE acts like a capacitor when $n=1$ and a resistor when $n=0$. The index $Y_{0}$ equals the admittance of CPE at the angular frequency $\omega=1 \mathrm{rad} / \mathrm{s}$.

To estimate whether the elements of the equivalent circuit differed depending on the type of hydrogel, repeated measures analysis of variance (rANOVA) was used. Then, to determine which groups in the sample differed, a post-hoc test, namely, Tukey's honest significant difference (HSD), was performed after the rANOVA.

\subsection{CIC}

The cathodic first (CF) biphasic constant-current pulse was applied between the evaluation electrode and the CE. The electrode potential of the evaluation electrode with respect to the RE was observed through the unity gain buffer amplifier using an oscilloscope (Wave Surfer 424, Teledyne LeCroy) (Fig. 6). The ground of the oscilloscope was isolated from the solution system by a

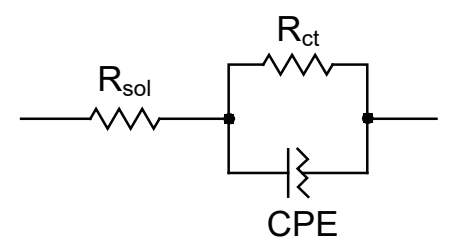

Fig. 5. Equivalent circuit model of the $\mathrm{Pt}$ stimulation elctrode $\left(R_{\mathrm{sol}}\right.$ : solution resistance, $R_{\mathrm{ct}}$ : charge transfer resistance, CPE: constant-phase element).

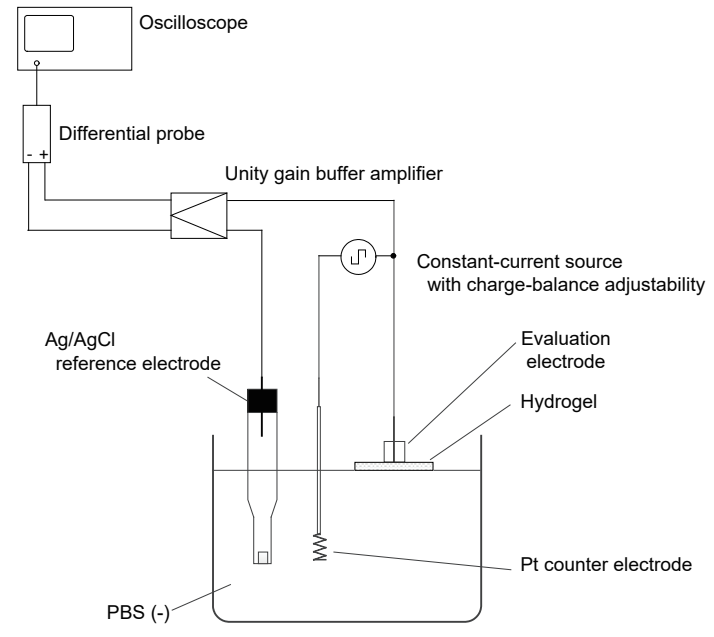

Fig. 6. Schematic view of the CIC measurement system. 
differential probe (N2791A, KEYSIGHT). The electrode potentials immediately after the current was stopped were truly the maximum polarization potentials (MPPs) without ohmic drops. The maximum allowable current $I_{\max }$ for the most-negative MPP, $E_{\mathrm{mc}}$, and the most-positive MPP, $E_{\operatorname{ma}}$, that did not exceed the water window was measured (Fig. 7). The water window was determined from the $\mathrm{CV}$ results as described above. The $\mathrm{CIC}$ was calculated by dividing the injectable charge at the time by the geometric surface area. To estimate whether the CICs differed depending on the type of hydrogel, rANOVA was used. Then, to determine which groups in the sample differed, Tukey's HSD was performed after rANOVA.

\section{Results}

Table 1 shows the water content ratio and $\mathrm{pH}$ of the hydrogels. The water content ratio of the $\mathrm{NC}$ gel was higher than that of the OR gel. There was no significant difference in $\mathrm{pH}$ between the hydrogels.

Typical CV results are shown in Fig. 8. There was no significant difference in the shape of the $\mathrm{CV}$ or in the range of the water window between the hydrogels. Therefore, the water window for the CIC measurements was determined to equal the theoretical value of PBS $(-0.6$ to $+0.8 \mathrm{~V})$.

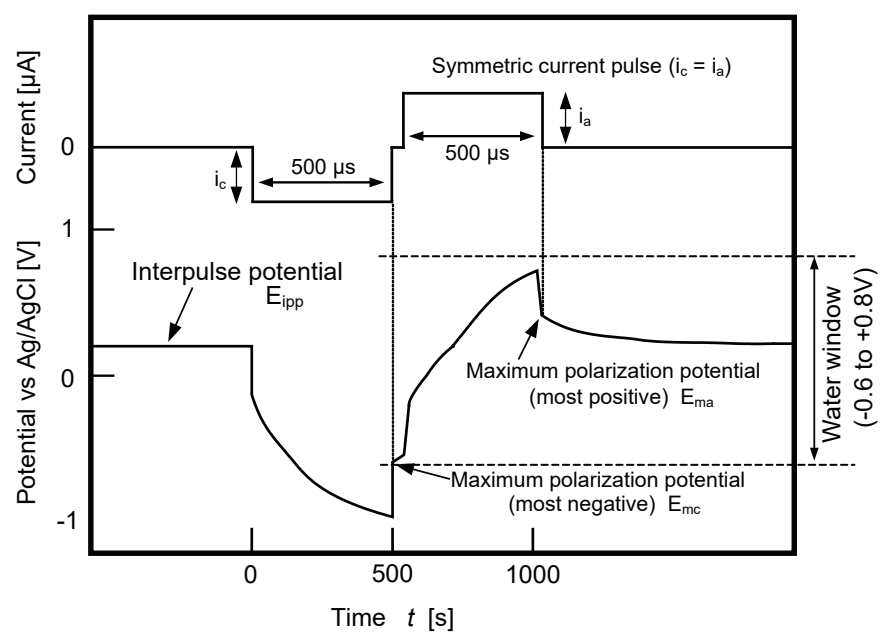

Fig. 7. Schematic view of the potential transient of the stimulation electrode in response to a biphasic symmetric current pulse. The CIC is defined as the maximum injectable charge without exceeding the water window (typical value: -0.6 to $+0.8 \mathrm{~V}$ vs $\mathrm{Ag} /$ $\mathrm{AgCl})$.

Table 1

Water content ratio and $\mathrm{pH}$ of the hydrogels.

\begin{tabular}{lccc}
\hline & $\begin{array}{c}\text { Water content } \\
\text { ratio }(\mathrm{wt} \%)\end{array}$ & $\mathrm{pH}$ & $\begin{array}{c}\text { Temperature } \\
\left({ }^{\circ} \mathrm{C}\right)\end{array}$ \\
\hline PBS (-) & - & 7.41 & 18.5 \\
NC gel & 96.7 & 7.47 & 19.6 \\
OR gel & 93.4 & 7.19 & 21.3
\end{tabular}

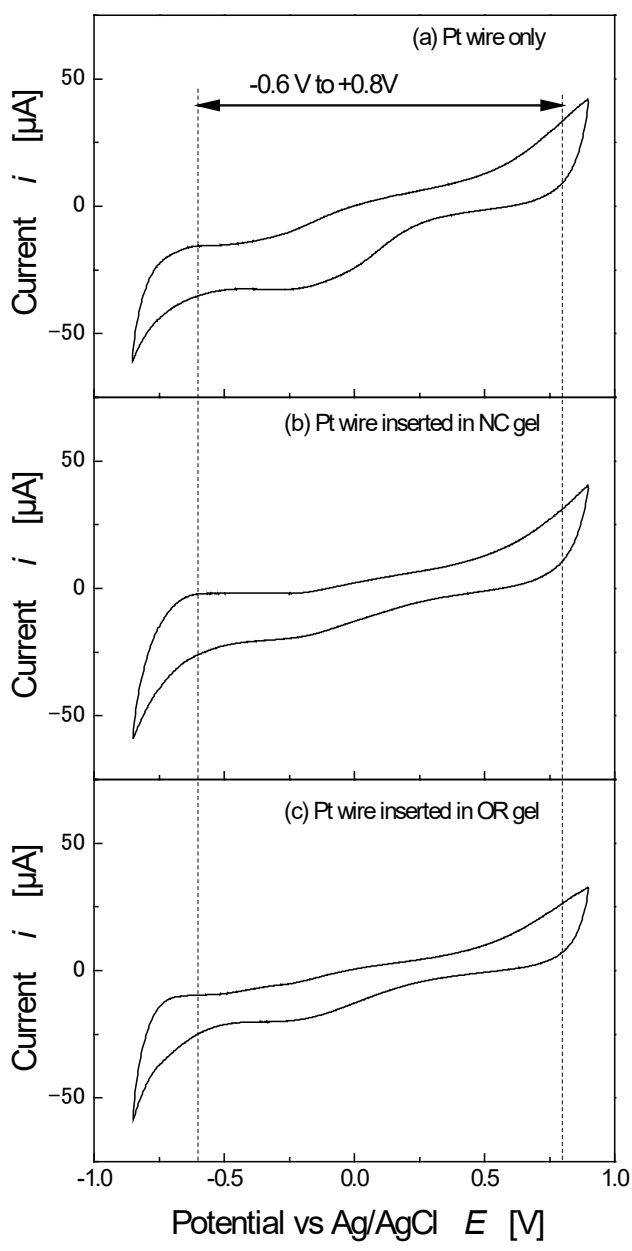

Fig. 8. Typical $\mathrm{CV}$ of the bare $\mathrm{Pt}$ wire and the $\mathrm{Pt}$ wire inserted in the hydrogels. 
Typical EIS results of the Pt disk electrodes and the glass-sealed Pt electrodes are shown in Figs. 9 and 10, respectively. The plotted points show the actual measured value, and the lines are the simulation results from the equivalent circuit analysis. The simulations agree very well with the
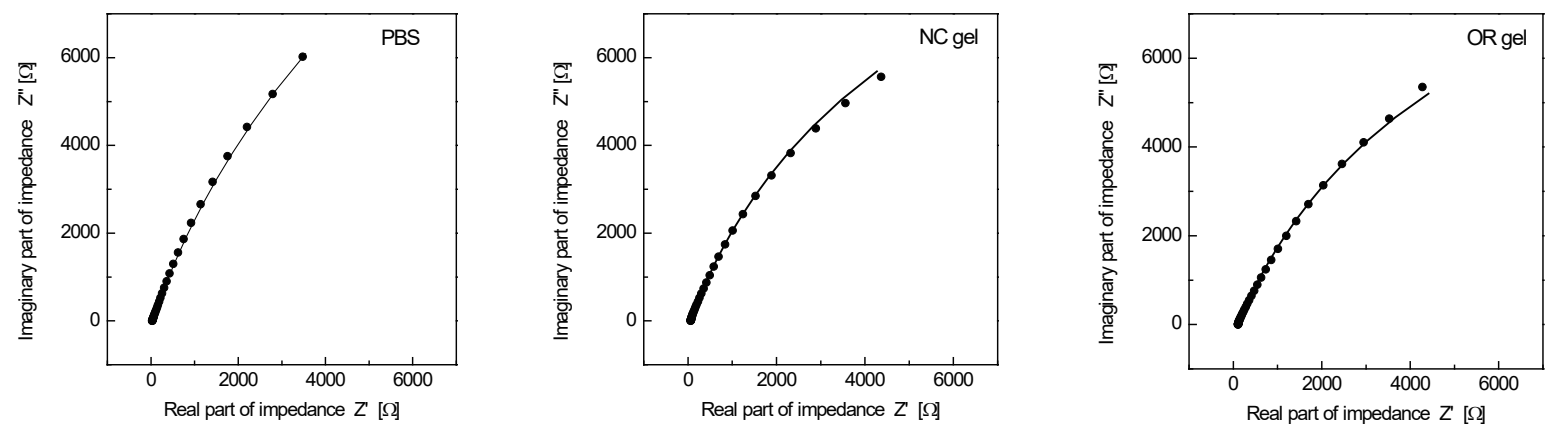

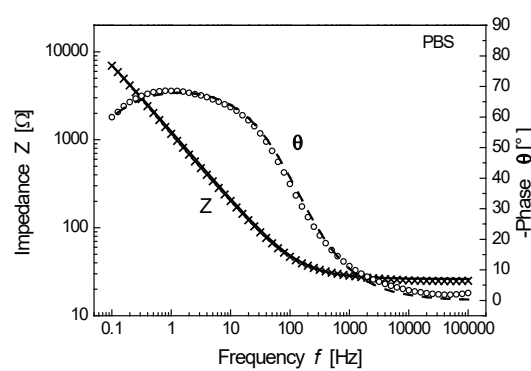

(a)

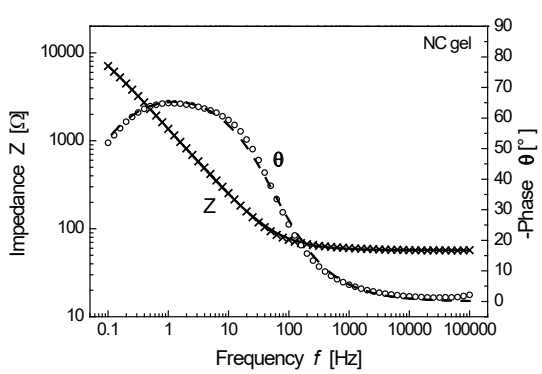

(b)

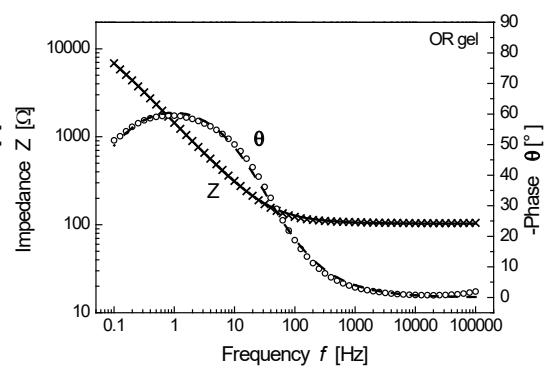

(c)

Fig. 9. Typical EIS results and equivalent circuit analysis of the Pt disk electrode. (a) PBS. (b) NC gel. (c) OR gel. The plotted points show the actual measured values. The lines are the fitted results of the equivalent circuit analysis.
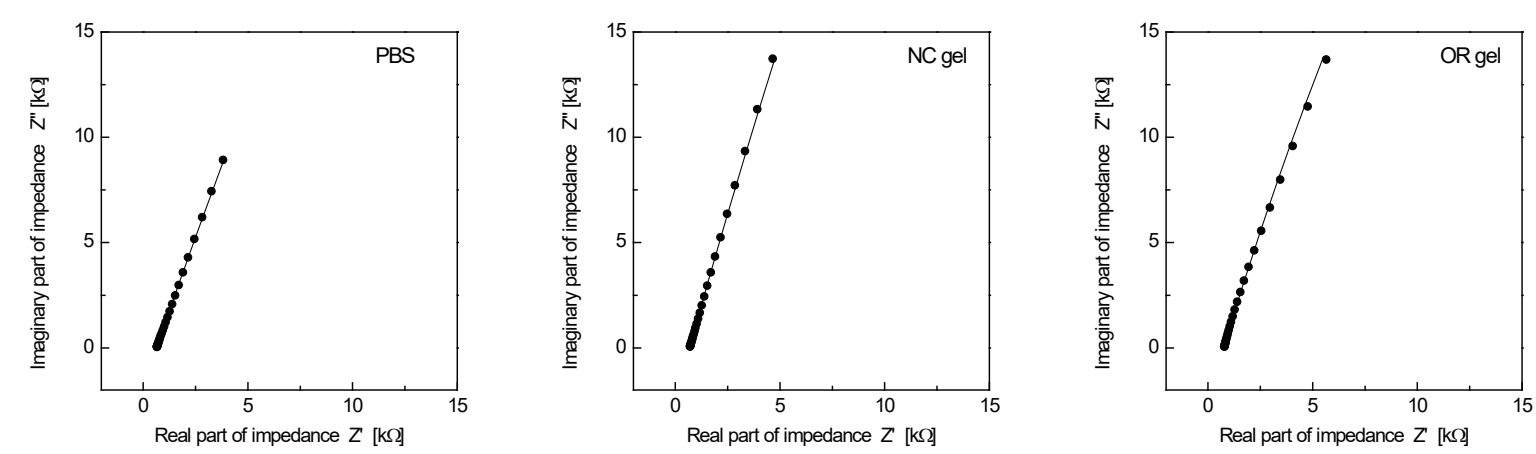

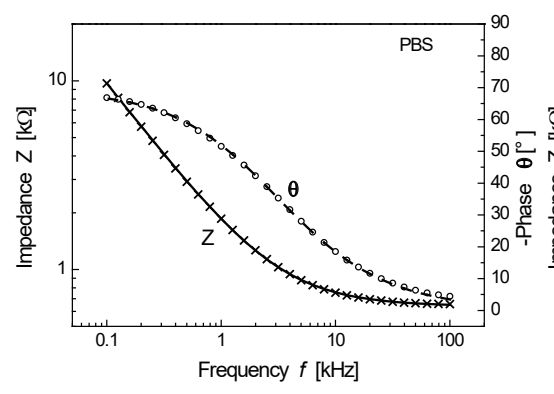

(a)

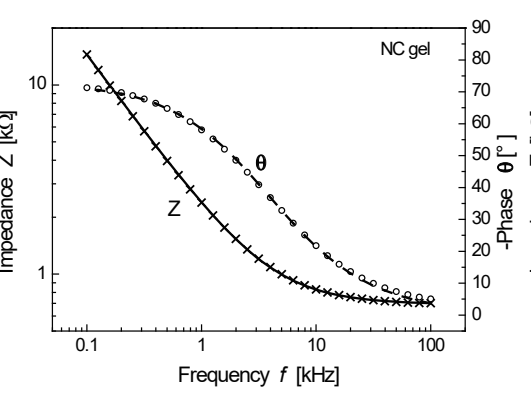

(b)

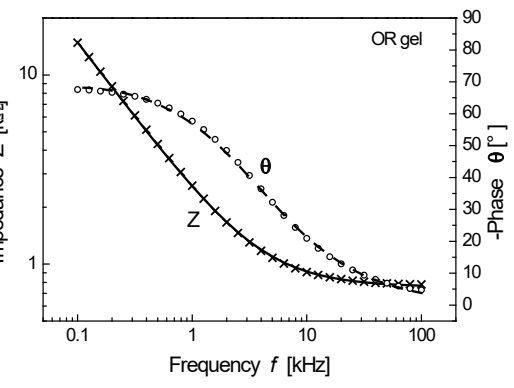

(c)

Fig. 10. Typical EIS results and equivalent circuit analysis of the glass-sealed Pt electrode. (a) PBS. (b) NC gel. (c) OR gel. The plotted points show the actual measured values. The lines are the fitted results of the equivalent circuit analysis. 
experiment. The means and standard deviations (SDs) for the equivalent circuit constants of the simulations are shown in Tables 2 and 3, respectively. The differences in $Y_{0}, n$, and $R_{\mathrm{ct}}$ between the hydrogels were not statistically significant at the 0.05 probability level according to the rANOVA results. The difference in $R_{\text {sol }}$ between the hydrogels was statistically significant at the 0.01 probability level (disk electrode: $F=68.1, p=0.0008$; glass-sealed electrode: $F=31.7, p=0.0035$ ). The difference in $R_{\text {sol }}$ for the Pt disk electrodes was statistically significant between all conditions, and that of the glass-sealed Pt electrode was statistically significant between the conditions with gel and PBS at the 0.05 probability level according to the results of Tukey's HSD (Fig. 11). The $R_{\text {sol }}$ value of the OR gel was the largest, and the $R_{\text {sol }}$ value of the NC gel was the second largest.

Tables 4 and 5 summarize the CIC measurement results. The difference in the CIC for the Pt disk electrode with a large surface area was not statistically significant at the 0.05 probability level

Table 2

EIS simulation results of the Pt disk electrode.

\begin{tabular}{lcccc}
\hline & $R_{\mathrm{sol}}(\Omega)^{* *}$ & $R_{\mathrm{ct}}(\mathrm{k} \Omega)^{\mathrm{NS}}$ & $Y_{0}(\mu \mathrm{S})^{\mathrm{NS}}$ & $n^{\mathrm{NS}}$ \\
\hline PBS & $30.7 \pm 6.16$ & $197 \pm 164$ & $92.2 \pm 24.1$ & $0.90 \pm 0.03$ \\
NC gel & $62.6 \pm 7.09$ & $674 \pm 820$ & $82.0 \pm 35.5$ & $0.89 \pm 0.03$ \\
OR gel & $106 \pm 8.33$ & $491 \pm 532$ & $66.3 \pm 13.9$ & $0.88 \pm 0.03$ \\
\hline
\end{tabular}

$n=3$. Values are mean $\pm \mathrm{SD}$.

$* *=$ highly significant $(p<0.01)$, NS $=$ not significant $(p>0.05$, rANOVA $)$.

Table 3

EIS simulation results of the glass-sealed Pt electrode.

\begin{tabular}{lllcc}
\hline & $R_{\text {sol }}(\Omega)^{* *}$ & $R_{\mathrm{ct}}(\mathrm{k} \Omega)^{\mathrm{NS}}$ & $Y_{0}(\mu \mathrm{S})^{\mathrm{NS}}$ & $n^{\mathrm{NS}}$ \\
\hline PBS & $719 \pm 85.0$ & $358 \pm 303$ & $315 \pm 287$ & $0.86 \pm 0.04$ \\
NC gel & $792 \pm 101$ & $403 \pm 261$ & $191 \pm 124$ & $0.88 \pm 0.05$ \\
OR gel & $849 \pm 85.0$ & $361 \pm 194$ & $190 \pm 141$ & $0.88 \pm 0.05$ \\
\hline
\end{tabular}

$n=3$. Values are mean $\pm \mathrm{SD}$.

$* *=$ highly significant $(p<0.01), \mathrm{NS}=$ not significant $(p>0.05, \mathrm{rANOVA})$.

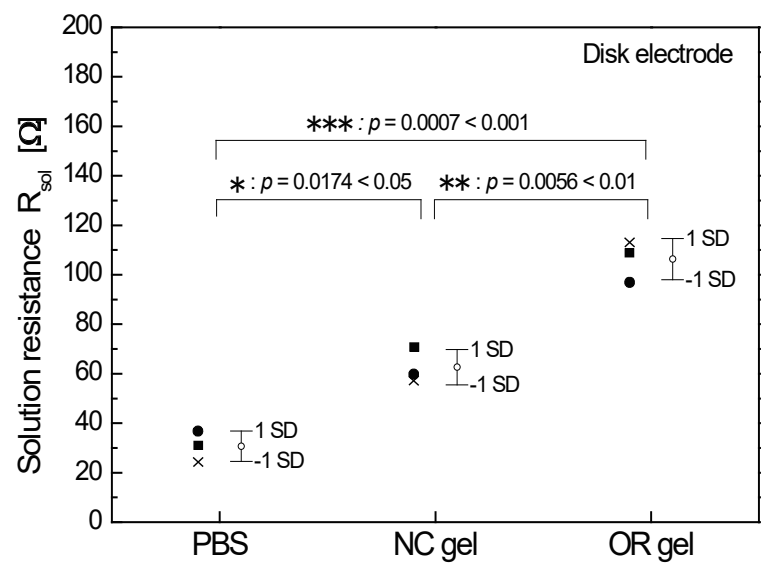

(a)

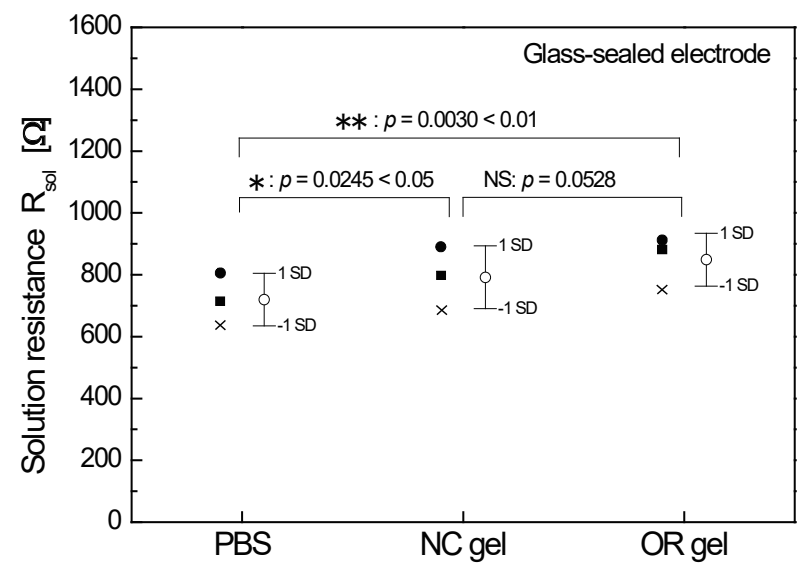

(b)

Fig. 11. Solution resistance of hydrogels. (a) Pt disk electrode. (b) Glass-sealed Pt electrode. The white circles are the mean values, and the bars show a range of $\pm 1 \mathrm{SD}$. $[*=$ significant $(p<0.05), * *=$ highly significant $(p<0.01)$, $* * *=$ highly significant $(p<0.0001)$, NS = not significant $(p>0.05)$ (Tukey's HSD)]. 
according to the rANOVA results. However, rANOVA revealed that the difference in the CIC for the glass-sealed Pt electrodes with microsurface areas was statistically significantly: $F=31.7, p=$ 0.00035. The CIC of the glass-sealed Pt electrode with the OR gel was significantly less than that of PBS at the 0.05 probability level according to results of Tukey's HSD (Fig. 12).

\section{Discussion}

The hydrogels used in the measurement had almost the same surface pH as PBS (-). The range of the water window depends on the $\mathrm{pH}$ of the solution. Therefore, no shift of the water window due to a difference in $\mathrm{pH}$ occurred. Actually, the $\mathrm{CV}$ results showed no significant difference. Therefore, the voltage range in which the Faradaic reaction occurred was not changed by the hydrogels.

Table 4

CIC measurement results of the Pt disk electrode.

\begin{tabular}{lccc}
\hline & Surface area $\left(\mathrm{cm}^{2}\right)$ & $I_{\max }(\mathrm{mA})$ & $\mathrm{CIC}\left(\mu \mathrm{C} / \mathrm{cm}^{2}\right)^{\mathrm{NS}}$ \\
\hline PBS & 0.785 & $77.6 \pm 12.5$ & $49.4 \pm 7.92$ \\
NC gel & 0.785 & $79.5 \pm 21.8$ & $50.6 \pm 8.03$ \\
OR gel & 0.785 & $79.3 \pm 30.5$ & $50.5 \pm 19.4$ \\
\hline
\end{tabular}

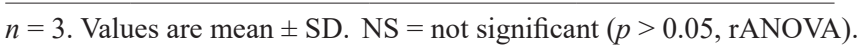

Table 5

CIC measurement results of the glass-sealed Pt electrode.

\begin{tabular}{lccc}
\hline & Surface area $\left(\mathrm{cm}^{2}\right)$ & $I_{\max }(\mathrm{mA})$ & CIC $\left(\mu \mathrm{C} / \mathrm{cm}^{2}\right)^{*}$ \\
\hline PBS & $1.96 \times 10^{-3}$ & $238 \pm 44.7$ & $60.7 \pm 11.4$ \\
NC gel & $1.96 \times 10^{-3}$ & $206 \pm 26.5$ & $52.5 \pm 6.75$ \\
OR gel & $1.96 \times 10^{-3}$ & $142 \pm 22.9$ & $36.1 \pm 5.84$ \\
\hline$n=3$. Values are mean \pm SD. ${ }^{*}=$ & significant $(p<0.05$, rANOVA $)$.
\end{tabular}

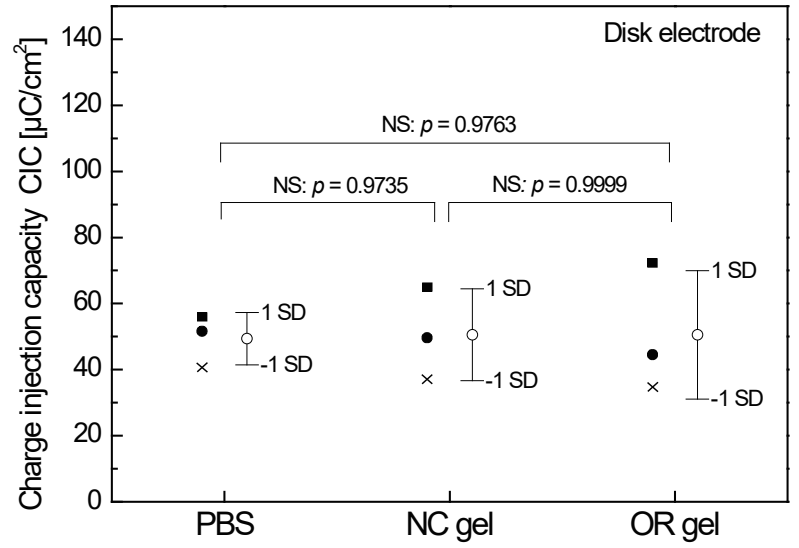

(a)

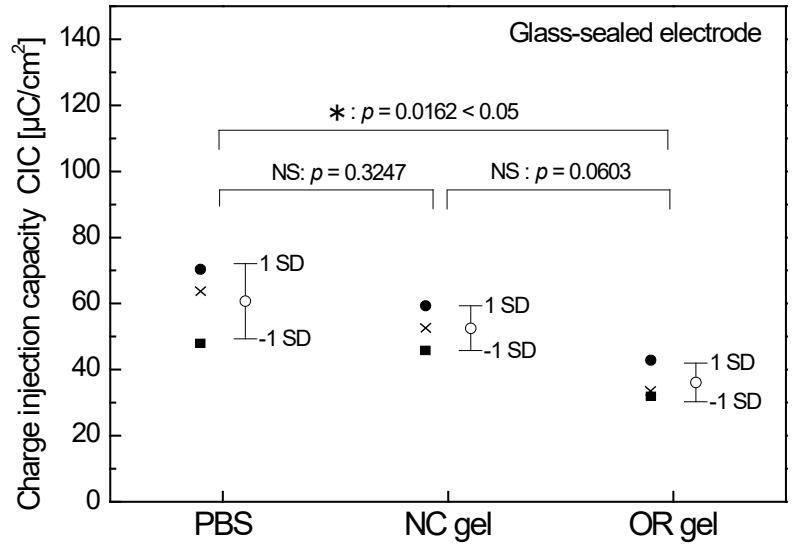

(b)

Fig. 12. CIC of hydrogels. (a) Pt disk electrode. (b) Glass-sealed Pt electrode. The white circles are the mean values, and the bars show a range of $\pm 1 \mathrm{SD}$. [ ${ }^{*}=$ significant $(p<0.05), \mathrm{NS}=$ not significant $(p>0.05)$ (Tukey's HSD)]. 
According to the EIS results, the CPE of the electrodes in the measurement functioned almost like a capacitor because the index $n$ of the CPE was approximately equivalent to 1 . Therefore, $Y_{0}$ was almost equivalent to the value of the double-layer capacitance $C_{\mathrm{dl}}$, where $Y_{0}$ and $R_{\mathrm{ct}}$ depend largely on the surface conditions of the electrode. The discharge and charge of $C_{\mathrm{dl}}$ are less affected by the existence of the hydrogels because the difference in the characteristics between the electrodes is larger than that between the conditions with and without a gel. The values of $R_{\text {sol }}$ were significantly different in the order of OR gel $>\mathrm{NC}$ gel $>$ PBS. These values were considered to be reflections of the water content ratio.

The difference in CIC of the Pt disk electrodes was not statistically significant. The electrode with a large surface area has a large $C_{\mathrm{dl}}$. Therefore, the amount of charge used for discharge and charge to $C_{\mathrm{dl}}$ was vastly the majority of the charge compared with the amount of charge required for the electrode reaction during the application of a constant-current pulse. It has been suggested that the lack of a significant difference in the CICs of the Pt disk electrodes is a reflection of the lack of a significant difference in the CPE. A significant difference in CICs of the microelectrodes, such as the glass-sealed Pt electrode, could be observed because of the effect of the charge used in the electrode reaction. A very small voltage was used in the EIS measurements to avoid an electrode reaction; however, a large voltage was applied in the CIC measurements. The depletion of ions and water necessary for the electrode reaction would decrease the CIC. The amount of ions and water is a reflection of the water content ratio. Therefore, it was considered that the water content ratio affects the CIC. Moreover, the CICs of the NC gel and PBS were equal to the typical CIC value of Pt in PBS.(12) We confirmed that the performance of the Pt electrode is unaltered by the NC gel coating in vitro. Additionally, the negative charge of synthetic hectorite has no effect on the electrochemical behavior of the electrode.

\section{Conclusions}

The performance of a Pt electrode covered with an $\mathrm{NC}$ gel that has an almost $100 \%$ water content ratio is confirmed to equal that of electrodes without a gel in vitro. If the CIC of the electrode covered with the NC gel maintains this value via the absorption of extracellular fluid and the existence of sufficient ions and water around the electrode surface, the performance of the electrode in a living body could increase. Thus, achieving a DNI or BMI with a higher density electrode array could be expected.

Future works include the implantation of a stimulation electrode covered with an $\mathrm{NC}$ gel in a rabbit eye and in vivo CIC and EIS measurements. We have already fabricated an NC-gel-coated microelectrode array for implantation. Furthermore, we also confirmed that the difference in CIC for the microelectrodes was not statistically significant between the conditions with and without the NC gel. ${ }^{(13)}$ The possibility of realizing the expected in vivo performance of the stimulation electrode with an $\mathrm{NC}$ gel will be evaluated.

\section{Acknowledgements}

We wish to thank the timely help given by Yukari Nakano of Nidek Co., Ltd. in the CIC measurements. This research was supported by the Adaptable \& Seamless Technology Transfer Program through Target-driven R\&D (A-STEP) from the Japan Science and Technology Agency (JST). 


\section{References}

1 F. A. Mussa-Ivaldi and L. E. Miller: Trends Neurosci. 26 (2003) 329.

2 NIDCD Information Clearinghouse: NIDCD Fact Sheet Cochlear Implants, NIH Publication No. 11-4798 (2016).

3 T. Fujikado, M. Kamei, H. Sakaguchi, H. Kanda, T. Morimoto, Y. Ikuno, K. Nishida, H. Kishima, T. Maruo, K. Konoma, M. Ozawa, and K. Nishida: Invest. Opthalmol. Vis. Sci. 52 (2011) 4726.

4 U. A. Aregueta-Robles, A. J. Woolley, L. A. Poole-Warren, N. H. Lovell, and R. A. Green: Front. Neuroeng. 7 (2014) 57.

5 Y. Terasawa, H. Tashiro, A. Uehara, T. Saitoh, M. Ozawa, T. Tokuda, and J. Ohta: J. Artif. Organs 9 (2006) 263.

6 Y. Terasawa, H. Tashiro, K. Osawa, M. Ozawa, T. Noda, J. Ohta, and T. Fujikado: Invest. Opthalmol. Vis. Sci. 53 (2012) 5538.

7 H. Tashiro, Y. Terasawa, K. Osawa, M. Ozawa, T. Noda, J. Ohta, and T. Fujikado: Invest. Opthalmol. Vis. Sci. 53 (2012) 5518.

8 A. Richter, Y. Xie, A. Schumacher, S. Löffler, R. D. Kirch, J. Al-Hasani, D. H. Rapoport, C. Kruse, A. Moser, V. Tronnier, S. Danner, and U. G. Hofmann: Front. Neuroeng. 6 (2013) 119.

9 K. Haraguchi, T. Takehisa, and M. Ebato: Biomacromolecules 7 (2006) 3267.

10 K. Haraguchi: Polym. J. 43 (2011) 223.

11 A. J. Bard and L. R. Faulkner: Electrochemical Methods: Fundamental and Applications (John Wiley \& Sons, Inc., Hoboken, 2001) 2nd ed., p. 13.

12 S. F. Cogan: Annu. Rev. Biomed. Eng. 10 (2008) 275.

13 Y. Terasawa, H. Tashiro, Y. Nakano, K. Osawa, M. Ozawa, T. Noda, T. Tokuda, J. Ohta, and K. Haraguchi: Invest. Ophthalmol. Vis. Sci. 57 (2016) 3727. 